\title{
Unmasking the vulnerabilities of uninfected children exposed to HIV
}

\author{
Although programmes to reduce vertical transmission of HIV mean fewer children are acquiring \\ HIV, more needs to be done to understand the longer term outcomes of exposure, say Vundli \\ Ramokolo and colleagues
}

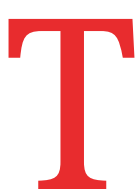
he number of children exposed to HIV who remain uninfected has been rising over the past two decades. In 2017, there were an estimated 14.8 million (uncertainty bounds 10.7-19.2 million) exposed but uninfected children, of whom $90 \%$ lived in sub-Saharan Africa. ${ }^{1}$ The number of uninfected children exposed to HIV in South Africa grew from one million in 2002 to more than three million in 2017 (fig 1). The increase is largely due to the success of public health programmes to prevent perinatal and postnatal vertical transmission of HIV. However, World Health Organization policy to provide lifelong antiretroviral treatment to all pregnant women with $\mathrm{HIV}^{2}$ has meant growing numbers of infected women conceive while taking antiretrovirals, leading to fetal exposure to antiretroviral drugs during the critical period of organogenesis in the first 10 weeks of a pregnancy.

Beyond preventing infants from acquiring HIV, it is important to ensure that exposed children also thrive. Research shows that uninfected children exposed to HIV are not only more likely to be have been born premature and small for their

\section{KEY MESSAGES}

- The number of uninfected children born to mothers with HIV has increased, reaching over 14 million in 2017

- Exposure to maternal HIV infection, maternal and infant antiretroviral drugs, and a household that includes people with HIV contribute to multiple health disparities

- Long term cohort studies are required to quantify which exposures drive outcome disparities and elucidate possible interventions

- Simultaneously, routine health services in high burden HIV settings should be equipped to provide early and timely identification and management of health disparities gestational age but also tend to have poorer growth and developmental outcomes than their unexposed counterparts. ${ }^{3}$ Thus, this vulnerable population needs close monitoring to quantify the short and long term effects of in-utero exposure to HIV and antiretrovirals so that effective interventions can be implemented to reduce any health or developmental disparities. Failure to invest in protecting the health of these children now has substantial implications for long term public health and human capital, particularly in settings with a high HIV burden. ${ }^{4}$

\section{How do we define exposure?}

In both research and operational settings, factors that potentially influence or cause ill health in uninfected children who have been exposed to HIV need to be well understood to prevent adverse outcomes. Furthermore, it has become increasingly important to clearly define the populations of uninfected exposed children, as variations in definitions limit comparability between studies and evidence synthesis. HIV exposed but uninfected typically refers to a child born to a mother with HIV, where the child has been exposed to HIV infection in utero or after delivery. However, there is much heterogeneity in this population because of changes in the HIV epidemic and antiretroviral drug regimens (table 1 ).

During the early stages of the HIV epidemic, some infants were born to women with HIV who did not receive antiretroviral drugs during pregnancy. These children do not have risks associated with intrauterine exposure to antiretroviral drugs but they were probably exposed to high levels of maternal HIV and systemic inflammation that could result in immune dysfunction. ${ }^{56}$ This has direct implications for the child's potential to survive and thrive because maternal viraemia is also associated with lower motor and expressive language scores $^{7}$ and poorer growth outcomes. ${ }^{8}$

One of the largest cohort studies of uninfected children who had been exposed to HIV, which included 3125 children, was initiated in Zimbabwe in 1997 before any antiretroviral drugs were available. ${ }^{5}$ Data from this study highlight the effects of HIV exposure alone on adverse birth outcomes such as preterm delivery, postnatal growth failure, and mortality, thereby emphasising the importance of primary HIV prevention and viral suppression for pregnant women with HIV.

Apart from HIV exposure, scale-up of more effective programmes to prevent infant HIV acquisition has created further heterogeneity, with some children exposed to HIV and antiretroviral drugs at the time of conception, others later in gestation, and yet others postnatally. Thus, depending on the timing of maternal diagnosis, an uninfected child's antiretroviral exposures may vary by the timing of initiation, duration, dose, and type of antiretroviral drugs. Recent data from a cohort study in South Africa report poorer growth among breastfed children exposed to HIV and antiretrovirals in utero compared with children who were not exposed to HIV. ${ }^{9}$ Additionally, antiretroviral drugs are now available as pre-exposure prophylaxis for women at high risk of HIV infection. Some women taking pre-exposure prophylaxis may conceive while taking antiretroviral drugs and continue them during pregnancy and lactation. ${ }^{10}$ Their children will be exposed to antiretroviral drugs but not to HIV in utero or after delivery. Monitoring systems are also needed for this group of children, whose numbers are likely to grow.

In addition to intrauterine exposures, it is equally important to quantify household, community, societal, and environmental factors such as food insecurity or poor water and sanitation. These factors inevitably contribute to adverse child health and developmental outcomes. Therefore, beyond the direct physiological exposure to maternal HIV and antiretroviral drugs through pregnancy and breastfeeding, a broader definition of HIV affected children should be considered (table 1). This would encompass children living in households or environments that currently include, or 


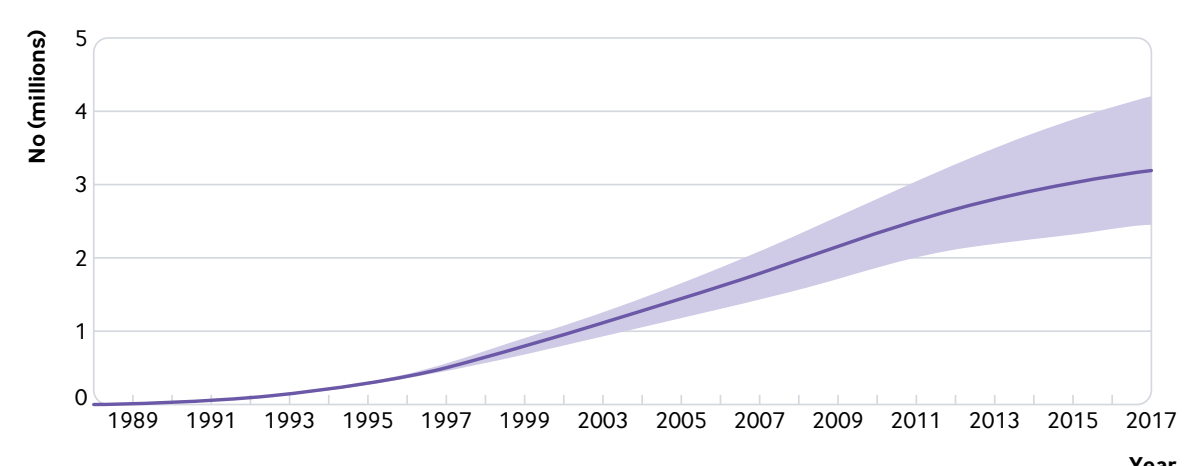

Fig 1 | UNAIDS estimates of the number of uninfected children exposed to HIV in South Africa. ${ }^{1}$ Solid line represents the point estimate with the uncertainty bounds in the shaded area

have included, a person with HIV. We refer to this population as environmentally HIV affected children.

\section{What research is needed to understand health outcomes?}

There is a paucity of long term follow-up data on uninfected children born to women with HIV, particularly in low and middle income countries with the highest HIV burdens, and particularly since increased coverage of antiretroviral drugs has meant many women are already taking antiretroviral drugs at conception. Now that antiretroviral regimens are recommended for use during pregnancy and breastfeeding, contemporaneous cohort studies and surveillance systems for exposed uninfected children are urgently needed.

Although modelled estimates, such as those recently published by UNAIDS, ${ }^{1}$ can describe and monitor trends in the uninfected exposed child population, more nuanced data from in-depth studies are required to understand risk factors and causal mechanisms for health and developmental deficits in these children. These studies require rigorous designs, such as long term prospective cohorts, with appropriately powered sample sizes that allow for complex analyses, and should be conducted in settings with the highest HIV prevalence.

\section{Strengthening routine follow-up}

In many low and middle income countries, children born to women with HIV have scheduled healthcare follow-up for immunisation and HIV testing until 18 months of age. More recently, the recommended follow-up period has been extended to 24 months for children who continue breastfeeding while their mothers receive antiretroviral treatment so that infection can be excluded once all HIV exposure

has ended. ${ }^{11}$ Concurrently, with research efforts to understand causal mechanisms and factors affecting the health, growth, and development of HIV exposed children, providers of routine health services should be aware of these children's vulnerability to known risks, including higher morbidity from infectious diseases, ${ }^{12}{ }^{13}$ to ensure early and timely identification and management of adverse outcomes.

Furthermore, uninfected children born to mothers with HIV are now entering adolescence and adulthood, requiring skills that enable independent functioning and contribution to society. A life course approach to identifying and caring for the most vulnerable exposed children through childhood, adolescence, and adulthood is thus urgently needed. Evidence indicates that children whose parents(s) have died from AIDS related diseases and those living in households with caregivers who have HIV experience vulnerabilities such as poverty have an increased risk of poor mental health. ${ }^{14}$ Thus, clinicians should be aware that some children may be environmentally affected by HIV, irrespective of their direct exposure; in this population of children interacting problems (family HIV infection, parental death from HIV, poverty, etc) increase the risk of poor health. ${ }^{15}$

Ultimately, research and health systems data may show that children with intrauterine exposure to HIV or early or long term antiretroviral drug exposure require specialised care and support, and these services may also be needed for a subset of children who are environmentally affected by HIV. Apart from routine health checks, most often completed at age 5 years, children generally interact with the healthcare system only when they are sick. They may re-enter the system as adolescents or adults seeking services such as family planning or maternity care. This presents a challenge for understanding the health and long term outcomes of HIV exposed children, since simply knowing that they did not acquire HIV during infancy is no longer a sufficient indicator of success.

Appropriately sized and structured routine child health data platforms, with systems to routinely analyse such data, will greatly facilitate evidence informed health policies and programmes so that resources and interventions can be suitably allocated and designed. Such platforms could also help answer important research questions relating to determinants of outcomes for children affected by HIV, particularly at the population level. Practical applications from such programmes could also include "trigger based" approaches where, for instance, specific events would trigger two key activities: an alert to the clinician for referral of the child for additional clinical assessments and care and reporting of the event into a database with a clearly defined denominator. ${ }^{16}$

One example of such systems is in the Western Cape Province of South Africa, where individual health records are linked through use of a unique identifier from birth into adulthood, ${ }^{17}$ importantly with linking of the mother-child dyad, ${ }^{18}$ to

\begin{tabular}{|c|c|c|c|}
\hline Exposure & $\begin{array}{l}\text { HIV exposed, } \\
\text { uninfected }\end{array}$ & $\begin{array}{l}\text { HIV unexposed, } \\
\text { ARV exposed * }\end{array}$ & $\begin{array}{l}\text { Environmentally } \\
\text { affected }\end{array}$ \\
\hline $\begin{array}{l}\text { Exposure to maternal HIV (varies by timing of maternal } \\
\text { infection, drug type, maternal viral load, and disease } \\
\text { stage) }\end{array}$ & Yes & No & No \\
\hline $\begin{array}{l}\text { Exposure to maternal antiretrovirals (varies by timing of } \\
\text { initiation, drug type, dose, and duration) }\end{array}$ & Yes & Yes & No \\
\hline $\begin{array}{l}\text { Exposure to infant antiretrovirals (varies by timing of } \\
\text { initiation drug type, dose, and duration) }\end{array}$ & Yes & No & No \\
\hline Exposure to household containing people with HIV & Yes & Maybe & Yes \\
\hline $\begin{array}{l}\text { Ubiquitous environmental exposure: poverty, poor water } \\
\text { and sanitation, food security, etc }\end{array}$ & Maybe & Maybe & Yes \\
\hline
\end{tabular}


track population outcomes. This approach uses the available detailed clinical data systems to improve individual clinical care and for public health surveillance, and provides insights on the risk factors needed to develop future public health interventions. ${ }^{19}$ Expanding this system to other parts of the country will require investments in infrastructure and human capacity, mechanisms to improve data quality, and standardisation and interoperability to ensure data can be transferred between new and existing software. ${ }^{18}$

\section{Multisectoral and ethical considerations}

Over $90 \%$ of uninfected children exposed to HIV live in countries with overburdened healthcare systems with competing priorities and a quadruple burden of disease, including communicable, noncommunicable, perinatal and maternal, and injury related events. ${ }^{20}$ The increasing evidence that a subset of uninfected children exposed to HIV and antiretrovirals have poorer neurodevelopmental outcomes than unexposed children, ${ }^{21}$ substantiates the urgent need for a multisectoral approach. For example, we need to track educational outcomes at population level by a child's HIV exposure status. However, careful thought and planning are necessary to ensure that applicable exposures or interventions are adequately captured to account for differences in academic performance. Such a strategy may necessitate disclosing HIV exposure status to the child or to key staff, such as healthcare providers and educators charged with monitoring outcomes or providing interventions to ensure the child thrives.

Although documenting children's in-utero exposure to HIV and antiretrovirals has benefits for healthcare and educational purposes, other aspects need consideration. Do the benefits of disclosure, beyond the breastfeeding period, outweigh or balance potential harms such as stigma or trauma? Does this impinge on the mother's or child's right to privacy? Some would argue that disclosing the mother's HIV status to healthcare providers during breastfeeding is justified because interventions that prevent transmission through breastmilk can be implemented and modified during this period, but that disclosure should stop with breastfeeding cessation, since the child is no longer at risk of vertical transmission. ${ }^{22}$ Others argue that children born to mothers with HIV, as well as those with environmental exposure, are at risk throughout their lifetime and thus, based on article 24 of the International Convention on the Rights of the Child, ${ }^{23}$ their healthcare providers need to be aware of their HIV or antiretroviral exposure throughout their lives.

Research is needed to understand the mechanisms leading to short and long term health and developmental disparities in uninfected children born to mothers with HIV. In high burden HIV settings, long term follow-up of HIV affected children to track vulnerabilities and monitor morbidity, quality of life, and mortality will require novel and collaborative approaches, such as harmonised data collection with data pooling for analyses between countries, recognising that these settings already have an overburdened health sector. Furthermore, strategies such as the promotion of timely capturing of complete and accurate facility level data and the training of data stewards will need to be adopted to strengthen the quality of routine data for effective monitoring. As healthcare providers and educators, our responsibility is to ensure that these children not only survive but also thrive, achieving optimal productivity in adolescence and adulthood.

Competing interests: We have read and understood BMJ policy on declaration of interests and declare no competing interests. This article was funded by the South African Medical Research Council and Unicef. Research reported in this publication was also supported by the Fogarty International Center of the National Institutes of Health under Award Number 1K43TW010683 to ALS and the Eunice Kennedy Shriver National Institute of Child Health and Human Development under Award Number 1R21HD093531 to KMP. ALS receives salary support through the CIPHER Grantee Programme of the International AIDS Society (2017/518-SLO) while VR receives support from the SAMRC Intramural Postdoctoral Fellowship Programme. The content of this paper is the sole responsibility of the authors and does not necessarily represent the official views of the organisations and funders.

Contributors and sources: AEG is an epidemiologist with more than 15 years of experience in clinical care and research. She has led national surveys to monitor effectiveness of approaches for prevention of transmission. She conceptualised the paper and contributed to its direction and finalisation; VR is an epidemiologist with over a decade of experience researching outcomes of HIV exposed and unexposed children. She led the writing. ALS is a paediatrician and epidemiologist with more than a decade of experience in research with and clinical care of HIV exposed children. KMP is a paediatrician and internist and has been conducting clinical research in the health disparities of HIV and antiretroviral exposed but uninfected infants and children for over a decade. All authors contributed to the ideas in the paper and the direction of the paper. All authors read the paper, contributed to all drafts, and approved the final version of the paper.

Provenance and peer review Commissioned; externally peer reviewed.

This article is part of a series proposed by the South African Medical Research Council and commissioned by The BMJ. The BM/ retained full editorial control over external peer review, editing, and publication. Open access fees are funded by SAMRC.

Vundli Ramokolo, researcher ${ }^{1}$

Ameena E Goga, researcher ${ }^{1,2,3}$

Amy L Slogrove, researcher ${ }^{4}$

Kathleen M Powis, assistant professor ${ }^{5,6,7}$

${ }^{1}$ Health Systems Research Unit, South African Medical Research Council, South Africa

${ }^{2}$ Department of Paediatrics, University of Pretoria, South Africa

${ }^{3}$ HIV Prevention Research Unit, South African Medical Research Council, South Africa

${ }^{4}$ Department of Paediatrics and Child Health and Ukwanda Centre for Rural Health, Faculty of Medicine and Health Sciences, Stellenbosch University, Worcester, South Africa

${ }^{5}$ Massachusetts General Hospital, Departments of Medicine and Pediatrics, USA

${ }^{6}$ Harvard T H Chan School of Public Health, Department of Immunology and Infectious Diseases, USA

${ }^{7}$ Botswana Harvard AIDS Institute Partnership, Gaborone, Botswana

Correspondence to: V Ramokolo

vundli.ramokolo@mrc.ac.za

\section{() (1) $\Theta$ OPEN ACCESS}

This is an Open Access article distributed in accordance with the Creative Commons Attribution Non Commercial (CC BY-NC 4.0) license, which permits others to distribute, remix, adapt, build upon this work non-commercially, and license their derivative works on different terms, provided the original work is properly cited and the use is non-commercial. See: http:// creativecommons.org/licenses/by-nc/4.0/.

\section{Check for updates}

1 UNAIDS. Number of HIV-exposed children who are uninfected. 2018 http://aidsinfo.unaids.org/

2 World Health Organization. Consolidated guidelines on the use of antiretroviral drugs for treating and preventing HIV infection-recommendations for a public health approach. 2nd ed. World Health Organization, 2016

3 Evans C, Jones CE, Prendergast AJ. HIV-exposed, uninfected infants: new global challenges in the era of paediatric HIV elimination. Lancet Infect Dis 2016;16:e92-107. doi:10.1016/S14733099(16)00055-4

4 Slogrove AL, Johnson LFP, Powis KM. Population-level mortality associated with HIV exposure in HIV-uninfected infants in Botswana and South Africa: a model-based evaluation. J Trop Pediatr 2018. [Epub ahead of print.]

5 Evans C, Humphrey JH, Ntozini R, Prendergast AJ. HIVexposed uninfected infants in Zimbabwe: insights into health outcomes in the pre-antiretroviral therapy era. Front Immunol 2016;7:190. doi:10.3389/ fimmu.2016.00190

6 John CC, Black MM, Nelson CA3rd.

Neurodevelopment: the impact of nutrition and inflammation during early to middle childhood in low-resource settings. Pediatrics 2017;139(Suppl 1):S59-71. doi:10.1542/peds.2016-2828H le Roux SM, Donald KA, Kroon M, et al. HIV viremia during pregnancy and neurodevelopment of HIV-exposed uninfected children in the context of universal antiretroviral therapy and breastfeeding: a prospective study. Pediatr Infect Dis / 2018. [Epub ahead of print.]

8 Ramokolo V, Lombard C, Fadnes LT, et al. HIV infection, viral load, low birth weight, and nevirapine 
are independent influences on growth velocity in HIVexposed South African infants. J Nutr 2014;144:428. doi:10.3945/jn.113.178616

9 le Roux SM, Abrams EJ, Donald KA, et al. Growth trajectories of breastfed HIV-exposed uninfected and HIV-unexposed children under conditions of universal maternal antiretroviral therapy: a prospective study. Lancet Child Adolesc Health 2019;3:234-44. doi:10.1016/S2352-4642(19)30007-0

10 Seidman DL, Weber S, Cohan D. Offering preexposure prophylaxis for HIV prevention to pregnant and postpartum women: a clinical approach. J Int AIDS Soc 2017;20(Suppl 1):21295. doi:10.7448/ IAS.20.2.21295

11 World Health Organization, United Nations Children's Fund. Guideline updates on HIV and infant feeding: the duration of breastfeeding, and support from health services to improve feeding practices among mothers living with HIV. World Health Organization, 2016.

12 Slogrove AL, Goetghebuer T, Cotton MF, Singer J, Bettinger JA. Pattern of infectious morbidity in HIV-exposed uninfected infants and children. Front Immunol 2016;7:164. doi:10.3389/ fimmu.2016.00164

13 Powis KM, Slogrove AL, Okorafor I, et al. Maternal perinatal HIV infection is associated with increased infectious morbidity in HIV-exposed uninfected infants. Pediatr Infect Dis / 2019:38:500-2. doi:10.1097/INF.0000000000002253

14 Cluver L, Gardner F, Operario D. Poverty and psychological health among AIDS-orphaned children in Cape Town, South Africa. AIDS Care 2009;21:73241. doi:10.1080/09540120802511885

15 Tsai AC, Mendenhall E, Trostle JA, Kawachi I. Cooccurring epidemics, syndemics, and population health. Lancet 2017;389:978-82. doi:10.1016/ S0140-6736(17)30403-8

16 Williams PL, Seage GR3rd, Van Dyke RB, et al, Pediatric HIV/AIDS Cohort Study. A trigger-based design for evaluating the safety of in utero antiretroviral exposure in uninfected children of human immunodeficiency virus-infected mothers. Am J Epidemiol 2012;175:950-61. doi:10.1093/aje/ kwr401

17 Rice B, Boulle A, Baral S, et al. Strengthening routine data systems to track the HIV epidemic and guide the response in sub-Saharan Africa. JMIR Public Health Surveill 2018;4:e36. doi:10.2196/ publichealth.9344

18 Osler M, Hilderbrand K, Hennessey C, et al. A three-tier framework for monitoring antiretroviral therapy in high HIV burden settings. J Int AIDS Soc 2014;17:18908. doi:10.7448/ IAS.17.1.18908
19 Birkhead GS, Klompas M, Shah NR. Uses of electronic health records for public health surveillance to advance public health. Annu Rev Public Health 2015;36:345-59. doi:10.1146/annurevpublhealth-031914-122747

20 Mayosi BM, Flisher AJ, Lalloo UG, Sitas F, Tollman SM, Bradshaw D. The burden of non-communicable diseases in South Africa. Lancet 2009;374:934-47. doi:10.1016/S0140-6736(09)61087-4

21 McHenry MS, McAteer Cl, Oyungu E, et al. Neurodevelopment in young children born to HIV-infected mothers: a meta-analysis. Pediatrics 2018;141:e20172888. doi:10.1542/ peds.2017-2888

22 Klitzman R, Mellins CA, Philbin MM, Abrams EJ, Remien RH. Ethical and psychosocial considerations in informing HIV-exposed uninfected children that they were exposed to HIV and antiretroviral medications in utero. Am J Public Health 2016;106:1390-6. doi:10.2105/ AJPH.2016.303257

23 United Nations General Assembly. Convention on the rights of the child. 1989 https://www.ohchr.org/en/ professionalinterest/pages/crc.aspx

Cite this as: BMJ 2019;366:14479

http://dx.doi.org/10.1136/bmj.l4479 